\title{
Fabrication and Design of Dendrimers for Cancer Chemotherapy
}

\author{
*Oyeniyi, Y.J. and Abdurahma , A. \\ Department of Pharmaceutics and Pharm.Microbiology, \\ Faculty of Pharmaceutical Sciences, Usmanu Dan Fodiyo University, Sokoto.
}

*Corresponding Author’s Contacts: drjimioyeniyi@gmail.com; +2348033472945

\begin{abstract}
Since the discovery of dendrimers in 1978, it has received serious attention as a drug carrier polymer most especially in cancer chemotherapeutics where precision and targeted delivery of drug to tumor cells is most desirable. Dendrimers are mostly synthetic, hyper-branched, tree-like globular, nano-sized polymers with excellent physicochemical properties that can be utilized in the formulation, design and delivery of drugs, vaccine and genes to specific receptors in the body. This review focused on the synthesis, types and applications of dendrimers in the delivery of cytotoxic drugs. The review shows that in the last decade, dendrimers have proved to be promising nanocarriers for various drugs including antiinflammatory, antimicrobial, and anticancer drugs. The application of dendrimers as scaffolds of prodrugs is particularly interesting. Dendrimers are relatively more stable compared with other nano drug carriers and are suitable in formulating drugs for different routes of administration. As more and newer dendrimers are introduced into the market, they will have increasing role in therapeutic delivery of drugs, vaccines and gene.
\end{abstract}

Key words: Dendrimers, Targeted drug delivery and Cancer chemotherapy

Introduction

Dendrimers are three dimensional polymers with unique features such as, hyper-branched, tree- like, globular structure, nanoscopic size, narrow polydispersity index, and excellent controlled molecular structure. Dendrimers are particularly interesting as they are relatively more stable when compared to other nano drug carriers. They also feature prominently in the design and fabrication of targeted drug delivery systems, which selectively deliver therapeutic agents to receptor sites and organs in the body. Dendrimer architecture can be distinguished easily from other nanoscopic polymers as they possess multiple functional groups at the periphery and interior lipophilic cavity which invariably contribute to the increasing solubility of hydrophobic drugs (Kesharwani et al., 2014). Formulation possibilities such as incorporation of both lipophilic and hydrophilic drugs substances within the same dendrimer and conjugation of drug molecule with the dendrimer peripheral multi functional groups are just but few of the numerous advantages offered by dendrimer based nanopharmaceuticals (Bhadra et al., 2005).
The first dendrimer was synthesized in 1978, and the word dendrimer originated from two Greek words, "Dendron", meaning tree and "meros" meaning part. Dendrimers can also be defined as synthetic, biodegradable and biocompatible macromolecules characterized by high branching points, three dimensional globular shape, monodispersity and nanometric size range (Kono et al.,1999; Dutta et al., 2006). There are three basic architectural components of a dendrimer as shown in Figure 1, (i) Interior core (ii) Interior layers composed of repeating units radically attached out of interior core (also refers to as the generations (iii) Exterior (terminal functionality) attached to outermost interior generation, the ligands (Surendra et al.,2014).

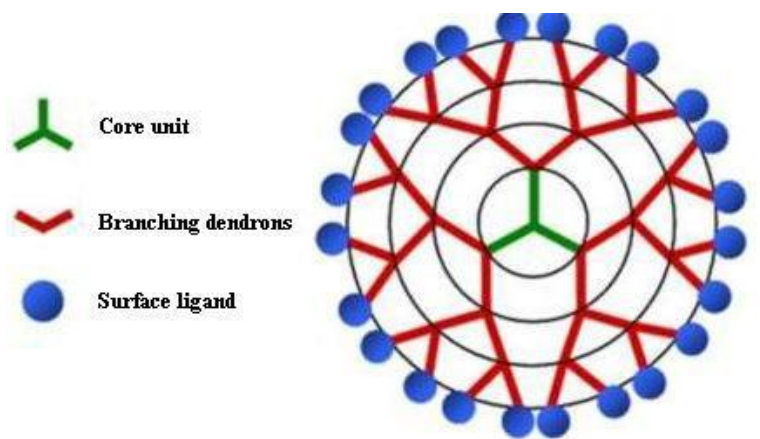

Figure 1: Basic structure of dendrimers 


\section{Methods of fabricating dendrimers}

The first synthesized dendrimer was polyamidoamines (PAMAM), also known as Starbus $t^{\circledR}$ dendrimers. The term Starbust ${ }^{\circledR}$ is a trademark of the Dow Chemicals Company. Basically, there are two approaches that may be used to synthesize dendrimers; divergent and convergent methods. Divergent method starts from the central core and extends outward toward the periphery, i.e. the dendrimer grows outwards from a multifunctional core molecule. The core molecule reacts with monomer molecules containing one reactive and two dormant groups giving the first generation dendrimer. Higher generations with higher molecular weights can be generated by activating the new periphery of the molecule for reactions with more monomers. The stepwise reaction can be repeated to obtain dendrimers of different generations. The divergent approach is very useful in the production of large quantities of high generations of dendrimers. The problem of side reactions and incomplete reactions of the end groups leading to structural defects are common in divergent method, however these problems can be solved by addition of excess reagents. Purification may also become an issue at the end of the reaction (Pushkar et al., 2006; Sakthivel et al.,1998).

The convergent method uses a top-down approach; it starts from the periphery of dendrimer, and grows inward to the inner core. The convergent method was developed as a response to the weaknesses of the divergent synthesis. In the convergent approach, the dendrimer is constructed stepwise, starting from the end groups and progressing inwards. When the growing branched polymeric arms, called dendrons, are large enough, they are attached to a multifunctional core molecule. The convergent growth method has several advantages, among which are the simplicity of purification of the desired product and minimization of the occurrence of structural defects in the final structure. It is also possible to introduce subtle engineering into the dendritic structure by precise placement of functional groups at the periphery of the macromolecule. The convergent approach however, does not allow the formation of high generations dendrimers because of high incidence of steric problem, which occurs during the synthesis (Hawker and Frechet, 1990;Tomolia, et al., 2005).

Each method has distinct advantages and disadvantages, and the selection of an appropriate route to generate a desired dendrimer depends mainly on the desired structure and generation type. While the divergent method is suitable for constructing high generation dendrimers, the convergent method is only suitable for synthesis of low molecular weight dendrimers.

Moat commercially available dendrimers such as polyamidoamine (PAMAM), poly (propylene imine) (PPI), poly-L-lysine, melamine, poly (etherhydroxylamine) (PEHAM) poly (esteramine) (PEA) and polyglycerol are synthesized by divergent method and are explored today as drug delivery vehicles (Wolinsky and Grinstaff 2008; Mintzer and Grinstaff, 2011).

\section{Types of dendrimers}

\section{PAMAM dendrimer}

All the generations of polyamidoamines (PAMAM) dendrimers are synthesized by the divergent pathway, starting with ammonia or ethylenediamine as the initiator core reagents. PAMAM dendrimers are commercially available from $\mathrm{G} 1$ to $\mathrm{G} 9$. G1 also called generation one represent dendrimers with low molecular weight, while generation nine (G9) are high molecular weight dendrimers usually presented as methanol solutions (Hawker \& Frechet, 1990). The synthesis of PAMAM dendrimers starts with the Michael addition between the multifunctional central core (usually, ammonia or ethylenediamine) and methyl acrylate. The shape of these PAMAM dendrimers may vary with their generations. Low generation PAMAM dendrimers (G1-G3) have ellipsoidal shapes, whereas the high generations PAMAM, (G4-G10) have welldefined cavities and roughly spherical shapes (Kim et al., 1998). Generally, G3 PAMAM dendrimers are considered more suitable as potential nanocarriers for poorly water soluble drugs (Ooya et al., 2004). Amine terminated PAMAM dendrimers could be useful in transporting drugs across various biological membranes, possibly by paracellular and endocytostic pathways. Moreover, by optimizing the size and surface charge, these dendritic platforms can be developed into oral delivery system for some orally inactive cytotoxic drugs. The various generations of PAMAM dendrimers, their molecular weight and number of functional groups are shown in table1. 
Table 1: Generations of PAMAM, their molecular weight and number of functional group.

\begin{tabular}{ccc}
\hline Generation & $\begin{array}{l}\text { Molecular } \\
\text { weight }\end{array}$ & $\begin{array}{l}\text { Number of } \\
\text { functional } \\
\text { group }\end{array}$ \\
\hline G1 & 1430 & 8 \\
G2 & 3256 & 16 \\
G3 & 6909 & 32 \\
G4 & 14215 & 64 \\
G5 & 28826 & 128 \\
G6 & 58048 & 256 \\
G7 & 116,493 & 512 \\
G8 & 233,383 & 1,024 \\
G9 & 467,162 & 2,048 \\
\hline
\end{tabular}

\section{PPI dendrimers}

Poly (propylene imine) dendrimers are polyalkyl amines, having primary amines as end groups, the dendrimer interior consists of numerous tertiary tris-propylene imines. Just like PAMAM, PPI dendrimers are commercially available from $\mathrm{G} 1$ to $\mathrm{G} 5$ generations, and have found widespread applications in biomedical, cosmetic, food and pharmaceutical industries. It is widely available as Astramol ${ }^{\circledR}$.

PPI dendrimers closely resemble PAMAM dendrimers except that the repeating units may create an internal environment with different polarity. The alkyl chain in PPI makes the internal microenvironment less polar compare to PAMAM dendrimers which have amido group as the repeating units (RichterEgger et al, 2001).

Other dendrimers described in the literatures are Tecto, Multilingual and Frechet dendrimers. A dendrimer core with other functionally different dendrimers attached to the core such that each of the peripheral (attached) dendrimers perform different functions such as, tumor cell recognition, diagnosis of disease state, drug delivery, reporting outcomes of therapy is referred to
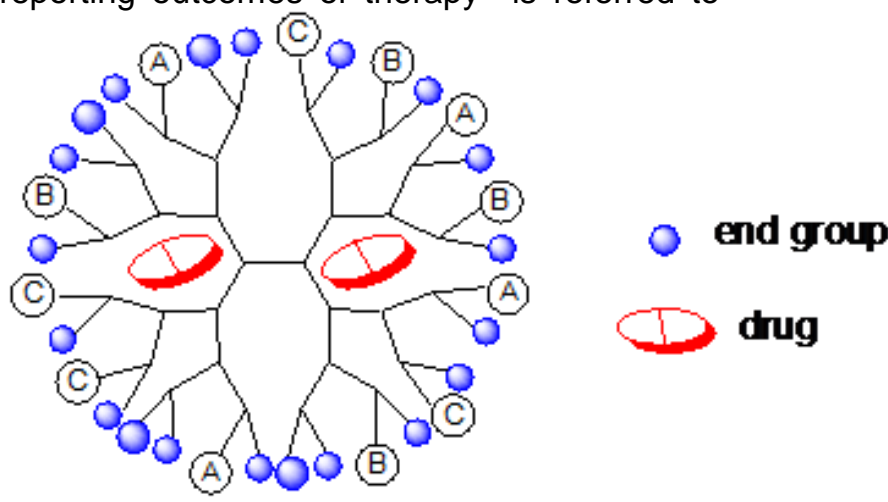

Figure 2: Encapsulation of drug into dendrimer core as Tecto dendrimer, while Multilingual dendrimers, surfaces contains multiple copies of the same functional groups.

Frechet-Type Dendrimers were first developed by Hawker and Frechet. The synthesis is based on poly-benzyl ether hyperbranched skeleton. These dendrimers usually have carboxylic acid groups as the surface groups, these carboxylic acid groups may serves as a good anchoring point for further surface functionalisation, and as polar surface groups to increase the solubility of this hydrophobic dendrimer type in polar solvents (Yiyun et al., 2008, Hawker, 2006).

\section{Designing a dendrimer based nanoparticle for drug delivery}

All PAMAM dendrimers, which fulfill most requirements for use in-vivo applications are being considered extensively for targeted drug delivery (Tomalia et al., 1985). PAMAM dendrimers are also being investigated as carriers in gene transfection (KukowskaLatallo et al., 1996). The internal tertiary amines of PAMAM dendrimers are available for acid-base interactions and hydrogen bonding as well as for other non-covalent interactions with encapsulated guest molecules, thus making the polymers effective agents for solubilizing hydrophobic drugs (Twyman, et al., 1999; Liu, et al., 2000).

Encapsulation is one of the methods used by various researchers to formulate dendrimer base drug delivery systems. Encapsulation of drugs uses the steric bulk of the exterior of the dendrimer and interactions between the dendrimer and the drug to trap the drug inside the dendrimer, Fig. 2.

Dendrimer-drug conjugates, however, have the drug attached to the exterior of the dendrimer. Most of these conjugates are prodrugs and are inactive or have decreased activity relative to the free drug.

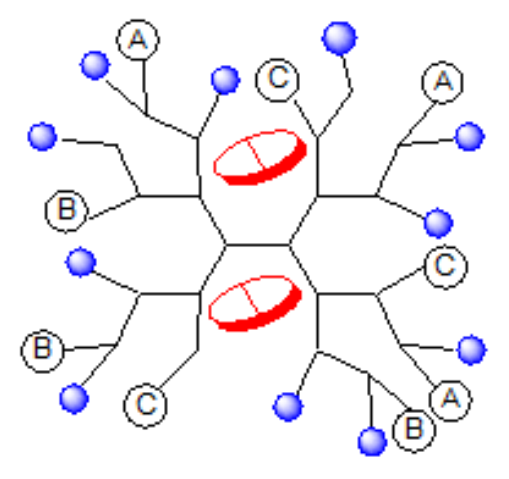


Conjugation refers to covalent attachment of free drug to the dendrimer.Dendrimer-drug conjugate offers two basic advantages as compared to entrapment,

- $\quad$ Prolong life time of drug in biological circulation.

- Improved drug stability

Drug-dendrimer conjugation is aimed at exploiting a well-defined multivalent aspect of dendrimers, which allows the attachment of drug molecules to its periphery. The resultant complexes are formed either due to the electrostatic interactions between the drug and dendrimer or conjugation of the drug to dendrimer molecule through electrostatic interactions (Zhou et al., 2005). Various ionizable drugs form complexes with the large number of ionizable terminal surface groups of dendrimers. For example, both primary amine and tertiary amine groups present on the surface of PAMAM dendrimers, offer potential sites for drug interactions. Adriamycin and methotrexate have been shown to form stable complexes through electrostatic interactions (Zhou et al., 2005). Moreover, the drugs can be covalently conjugated to dendrimers through some spacers that may include PEG, p-amino benzoic acid, p-amino hippuric acid and lauryl chains etc., or biodegradable linkages such as amide or ester bonds. This prodrug approach has been found to increase the stability of drugs and has affected their release kinetics significantly. Several researchers have successfully conjugated many anticancer drugs such as cisplatin, doxorubicin, epirubicin, methotrexate and paclitaxel with PAMAM dendrimers. The results have shown the enhanced solubility and controlled release of drugs from these complexes in comparison to the pure drug (Kaminskas et al., 2012; Gurdag, et al., 2006). Dendrimer-drug conjugates have been reported as a potential platform for a combine uses as diagnostic and therapeutic agents. Such combination of both diagnostic and therapeutic agent in a single dendrimer is termed theranostic drug delivery system.

\section{Problems associated with the administration of cytotoxic agents}

The success of all cytotoxic drugs depends on the consistent availability of adequate quantity of cytotoxic agents in various tumor cells. After the administration of conventional anticancer drugs, they are distributed blindly throughout the whole body via the bloodstream. They affect both malignant and rapidly dividing normal living cells of the bone marrow, lymphoid tissue, supermatogenic cells, fetus as well as hair follicles (Links et al., 1999). This account for severe side effects associated with the use of most convection cytotoxic drugs. Other problems associated with the use of cytotoxic agents are, high patient risks, repeated treatments, altered biodistribution of drug and the acquisition of multidrug resistance (MDR) by the cancer cells (Brigger et al., 2002). Since most cancer chemotherapies lack specificity and selectivity, incorporation of ligands on dendrimer surfaces is one of the methods utilized to effect target delivery of anticancer molecules to the tumor cells, thereby reducing adverse drug effects associated with the administration of some of these chemotherapeutic drugs (Jain 2005; Ould et al 2005; Kipp, 2004).

Acquisition of drug resistance by cancer cells is another factor. Resistance to cytotoxic agents could be acquired through both noncellular as well as cellular mechanism. The non-cellular resistance occurs due to poorly vascularized tumor regions which can effectively reduce drug access to the tumor and thus protect cancerous cells from cytotoxicity. The acidic environment in the tumors cells can also confer a resistance mechanism against alkaline cytotoxic drugs. These compounds undergo ionization, thereby preventing their diffusion across cellular membrane. High interstitial pressure and low microvascular pressure may also retard or impede extravasation of molecules.

The cellular mechanism of resistance involves over expression of the plasma membrane Pglycoprotein (P-gp), which is capable of repelling drugs from the tumor cells, thereby leading to a decreased cell sensitivity and intracellular accumulation of drugs. This is more pronounced with drugs which enters the cell by passive diffusion through lipid bilayer. Upon entering into cell, these drugs bind to Pgp, which forms transmembrane channels and uses the energy of ATP hydrolysis to pump these compounds out of cells (Krishna et al., 2000).

Co-administration of P-gp inhibitors with encapsulated anticancer drugs in nanoparticles have been proposed to prevent P-gp-mediated multi drug resistance (MDR) (Krishna et al., 2000). Other limitations include reduced potency observed, in some drugs after linkage to targeting moieties when targeting portion is not cleaved correctly 
(Krishna, et al., 2000). Rapid blood clearance by the reticuloendothelial system (RES) is another major problem in cancer therapeutics. The RES is part of the natural body defense mechanism of which monocytes and macrophages located in reticular connective tissue (the spleen), are responsible for attacking and removing cellular debris, pathogens and foreign substances from the bloodstream through phagocytosis. Formulation parameters such as particle size, hydrophobicity, surface charge and composition of the nano particle formulation influence the clearance profile of the delivery system. RES problem can be overcome by PEGylation (attachment of polyethylene glycol to the drug carriers). PEGylation has also been proposed for other delivery systems in order to reduce their clearance by reticuloendothelial system (Klibanov et al., 1990 and Blume et al., 1990).

\section{Tumor Targeting with dendrimer based drug delivery system}

Drug targeting is a concept that aims to directly deliver therapeutic agents to a specific site, cell type, tissue or organ in the body. This is extremely beneficial in cancer chemotherapeutics where cytotoxic drugs that kill tumor cells may also kill or adversely damage healthy living tissue as a result of indiscriminate distribution of drugs to different tissues and organs in the body.

In cancer therapy several approaches have been proposed to achieve selective delivery of drugs to tumor cells while sparing normal healthy cells. One method employs the enhanced permeability and retention (EPR) phenomenon in tumors where microvasculature system becomes more permeable and allows accumulation of drug molecules selectively in the cancer cells (Maeda et al., 2000; Ihre et al., 2002). In EPR targeting, since the therapeutic agents are selectively absorbed into the tumor cells, it effectively kills the cancer cells with minimal effect on normal healthy cells and tissues (Padilla De Jesus et al., 2002).

Another useful approach utilizes tumor cells specific antigens. Normally, tumor cells express or over-express unique receptors or antigens. Once identified, they can be targeted using specific antibodies, small molecules or peptides that can recognize and bind to these tumor expressed antigen. In this approach, therapeutic or bioactive agents are coupled directly to targeting moiety for selective delivery. However, there are limitations such as decreased solubility, and limited drug carrying capability, posed by some of these conjugates. There are ongoing investigations to design novel carrier systems that can overcome these problems, i.e. ensures longer circulation times and increased the potential to carry larger amounts of drug. One of the aims of this approach is to ensure that macromolecules that are used as drugs carriers remain soluble and bind effectively both therapeutic and targeting ligands until they reach the targeting sites. Any degradation of the carrier or premature release of free drug before reaching the desired location defeats the purpose of this delivery system. For this reason, a stable and reliable system is most desired. Nano-scale dendrimers have been proposed and investigated as an effective carrier for anti cancer drug targeting (Langer, 1990; Newkome, et al., 2001; Frechet and Tomalia, 2001).

\section{Some clinically useful dendrimer nano- formulations}

Dendrimers offer various formulation possibilities, among which is their ability to incorporate two or more drugs within the same polymer. Various generations of PAMAM dendrimers having PEG grafts were synthesized and evaluated for their ability to encapsulate two different anticancer drugs: Adriamycin (ADC) and methotrexate (MTX). It was shown that encapsulation efficiency increases with increases in both PAMAM generations and the molecular weight of the attached PEG. It was also inferred from the study that the increased encapsulation efficiency of MTX by dendrimer was due to acid-base interaction between MTX (acidic) and amino groups of dendrimer polymer (Kojima et al., 2000)

A PEGylated-PAMAM (G-4) formulation of 5fluorouracil (5-FU) was reported to reduce therapeutic side effects, normally experienced after administration of convectional 5fluorouracil. This formulation also display low hemolytic toxicity, high drug loading capacity, increased cytotoxicity and improved tumor cells permeability. It was also observed that after intravenous administration of different formulations of 5-FU to rats through the caudal vein, the maximum drug concentration $\left(\mathrm{C}_{\max }\right)$ from free drugs, nonPEGylated dendrimers, and PEGylated dendrimers, was 200-220, 2123 , and $6-7 \mathrm{mg} / \mathrm{mL}$, respectively. It was only in the PEGylated dendrimers formulation that the blood level of 5-FU was detectable for up to 12 $\mathrm{h}$ after the administration of the drug (Bhadra et al., 2003). 
A Fifth generation of PAMAM formulation of methotrexate was found to be more effective in killing tumor cells by conjugating MTX to PAMAM-fluorescein isothiocyanate -folic acid complex (PAMAM-FITC-FA) via amide and ester linkages. The conjugates were internalized in the KB cell line of human epidermoid carcinoma. The plain MTX was found to be less effective in killing tumor cells when compare to drug conjugates through ester linkages (Quintana et al., 2002).

Patri and coworkers synthesized G5 PAMAMFA-MTX complex. They however modified the surfaces of a second batch so that negatively charged (carboxylate) groups were obtained as terminal functionalities. Binding characteristic of all the modified dendrimers incorporating MTX with and without FA were performed on FR (+) KB cells. The results shows a decrease in non-specific interaction of the dendrimers as compared to amine terminated ones and a greater accumulation of the complex in FR $(+)$ cells, which is further supported by lack of cytotoxicity in $\mathrm{FR}(-)$ cells (Patri et al., 2005).

A conjugate of PAMAM dendrimers and cisplatin, (a potent anti-cancer drug with nonspecific toxicity and poor solubility) was equally investigated. The conjugate shows increased solubility, decreased systemic toxicity and enhanced permeation and retention properties (Malik et al., 1999). Intravenous administration of this conjugate to mice bearing B16F10 tumors was able to selectively accumulate cisplatin in solid tumors (50-fold increase compared to that achieved after intravenous administration of plain cisplatin at its maximum tolerated dose).

Cationic dendrimers were evaluated for Deoxyribonucleic acid (DNA), Ribonucleic acid (RNA) and microRNA (miRNA) delivery, and this has become routine tools in cell biology and many other biomedical fields.

It has been reported that intravenous administration of G9 PAMAM dendrimercomplex pCF1CAT plasmid into rats could result in high levels of gene expression in the lung tissues (Kukowska-Latallo et al., 1996).
In order to enhance the transfection efficiency and expression pattern of the dendrimer, a cyclodextrin was conjugated on the surface of G3 PAMAM dendrimer. And after the intravenous administration of this hybrid material and DNA complex into the rats, they found that the organ where the transgene dominantly expressed was in the spleen (Kihara et al.,2005).

Based on this study, mannose was conjugated to this hybrid material and this new multifunctional material was used as a new gene transfection agent. After the intravenous injection, the hybrid material bearing mannose ligands showed higher transfection activity in the kidney than dendrimer alone and the hybrid material without mannose ligands (Kukowska-Latallo et al., 1996 ; Kihara et al.,2005 ; Wada et al.,2003).

Various modified PPI dendrimers were evaluated as effective transfection agents for catalytic DNA enzymes. The results showed that intravenous administration of G4 PPI dendrimer-PEG conjugate and DNA complex into Nude mice could lead to a high gene transfection efficacy and nuclear uptake. Based on these results, it was concluded that dendrimers were promising gene vectors with higher capability to deliver gene into the liver, spleen, lung, kidney, and even the tumor at therapeutic levels, and the intravenous administration route may be more suitable (Tack et al., 2006).

Oral drug delivery studies using the human colon adenocarcinoma cell line (Caco-2) have indicated that low generation PAMAM dendrimers cross cell membranes, presumably through a combination of two processes; paracellular transport and adsorptive endocytosis. Remarkably, the P-glycoprotein efflux transporter does not appear to affect dendrimers, therefore drug dendrimer complexes are able to bypass the efflux transporter. PAMAM dendrimers conjugated with folic acid and fluorescein isothiocyanate were use for targeting and imaging tumor cells respectively (Barbara \& Maria, 2001, Rajeshbabu et al., 2010). Some clinically relevant dendrimer based anticancer drug formulations are shown in Table 2 . 
Table 2: Some commercially available dendrimer based anticancer formulations

\begin{tabular}{|lll|}
\hline Drugs & $\begin{array}{l}\text { Dendrimer } \\
\text { Polymer used }\end{array}$ & Therapeutic Improvement \\
\hline Methotrexate & G2.5 and G3 PAMAM & 24-fold increment in cytotoxicity \\
\hline Paclitaxel & $\begin{array}{l}\text { Polyglycerol } \\
\text { (G4 and G5) } \\
\text { G4 PAMAM }\end{array}$ & Increased aqueous solubility \\
& $\begin{array}{l}\text { Increased aqueous solubility } \\
\text { (glycerolsuccinic acid) }\end{array}$ & High cytotoxicity \\
\hline $\begin{array}{l}\text { 10-hydroxy } \\
\text { camptothecin }\end{array}$ & G4 PAMAM & Targeted drug delivery \\
\hline E-Fluorouracil & G4 and G5PAMAM & High loading capacity \\
\hline Doxorubicin & G4 PAMAM & Improved cytotoxicity \\
\hline Doxorubicin & G5 polylysine & Prolonged plasma exposure \\
& & and diminished drug toxicity \\
\hline
\end{tabular}

A G5 conjugate of FA-PAMAM-MTX was investigated for the targeted delivery of methotrexate, the conjugate demonstrated high specificity for KB cells over expressing folate receptors and showed sustained drug release (Patri et al., 2005)

The authors further conjugated the PhiPhiLux G1 D2, an apoptotic sensor to FA attached PAMAM dendrimers and this showed an enhanced fluorescence, attributed to successful delivery of drug with higher cell killing efficacy ( Myc et al., 2007). FA conjugated G5 PAMAM dendrimers successfully delivered 2 methoxyestradiol to targeted cancer cells, as confirmed by MTT assay (Wang et al., 2011). A folate functionalized PEG coated nanocarriers based on fourth generation diaminobutane PPI dendrimer was evaluated for targeted delivery of etoposide. The encapsulation of the drug inside the dendrimeric scaffold resulted in enhanced solubility of etoposide and the invitro release of encapsulated drug from this functional dendrimer was found to be sustained and comparable to the nonfunctionalized dendrimers. These folate PEGylated dendrimers exhibited specificity for folate receptors with low toxicity (Sideratou et al., 2010). Lastly, successful conjugation of FA to fifth generation PPI dendrimers was explored and its potential in targeted delivery of an anticancer drug doxorubicin was investigated. The results revealed that FA conjugated dendrimers displayed higher cell uptake in MCF-7 cancer cell lines and significantly reduced the toxicity (Gupta et al., 2010).

\section{Conclusion}

Potentials of dendrimers as vehicles for sitespecific delivery of anti-cancer drugs seems to be promising. Dendrimers will be expected to keep playing key role in biomedical, and pharmaceutical fields as they provide an excellent platform for drug conjugation and have the ability to encapsulate or bind differentyi and Abdurah drugs via several mechanisms such as physical encapsulation and electrostatic interaction. Their high costs, complex synthesis procedure and toxicity issues are however few drawbacks..

\section{Acknowledgement}

Special thanks to Prof (Dr.) Biswajit Mukherjee, Department of Pharmaceutical Technology, Jadavpur University, Kolkata, India for his useful discussions

\section{Conflict of Interest}

There is no conflict of interest regarding the manuscript.

\section{References}

Barbara, K. and Maria, B. (2001). Dendrimers: properties and application. Acta Biochimica Polonica, 48: 199-208. 
Bhadra, D., Bhadra, S., Jain, S. and Jain, N. K. (2003). A PEGylated dendritic nanoparticulate carrier of fluorouracil. Int J Pharm, 257:111-124.

Bhadra, D., Yadav, A.K.., Bhadra, S. and Jain, N.K. (2005). Glycodendrimeric nanoparticulate carriers of primaquine phosphate for liver targeting. Int $J$ Pharm., 295:221-233.

Blume, G. and Cevc, G. (1990). Liposomes for the sustained drug release in-vivo. Biochim Biophys Acta. 1029:91-97.

Brigger, I., Dubernet, C. and Couvreur, P. (2002). Nanoparticles in cancer therapy and diagnosis. Adv Drug Deliv Rev. 54:631-651.

Dutta, T., Aghase, H.B., Vijayarajkumar, P., Joshi, M. and Jain, N.K. (2006). Dendrosome-based gene delivery. Journal of Experimental Nanoscience, 1:235-248.

Gurdag, S., Khandare, J., Stapels, S., Matherly, L.H. (2006). Activity of dendrimer-methotrexate conjugates on methotrexate sensitive and resistant cell lines. Bioconjug Chem. 17:275-283.

Fre'chet, J.M.J. and Tomalia, D.A. (2001), Dendrimers and Other Dendritic Polymers, Wiley, West Sussex, UK. pp 225- 275.

Gupta, U., Dwivedi, S.K., Bid, H.K., Konwar, R. and Jain, N.K. (2010). Ligand anchored dendrimers based nanoconstructs for effective targeting to cancer Cells. Int J Pharm, 393: 185 $-196$.

Hawker, C.J. and Frechet, J.M.J. (1990). Preparation of polymers with controlled molecular architecture: A new convergent approach to dendritic macromolecules. J Am Chem Soc 112:7638-7647.

Hawker, C.J. (2006). Dendrimers: Novel polymeric nanoarchitectures for solubility enhancement. Biomacromolecules, 7: 649-658.

Ihre, H.R., De Jesus, O.L.P., Szoka, F.C. and Frechet, J.M.J. (2002). Polyester dendritic systems for drug delivery applications: design, synthesis, and characterization, Bioconjug. Chem. 13:443- 452 .

Jain, K. (2005). Nanotechnology based drug delivery for cancer. Technology in Cancer Research \& Treatment 4:407.

Kaminskas, L.M., McLeod, V.M., Kelly, B.D. and Sberna, G. (2012). A comparison of changes to doxorubicin pharmacokinetics, antitumor activity, and toxicity mediated by PEGylated dendrimer and PEGylated liposome drug delivery systems. Nanomedicine,8:103-111

Kim, Y.K. and Zimmerman, S.C. (1998). Applications of dendrimers in bioorganic chemistry. Curr Opin Chem Biol 2:733-742.

Kipp, J. (2004). The role of solid nanoparticle technology in the parenteral delivery of poorly water soluble drugs. International Journal of Pharmaceutics, 284:109-122.

Klibanov, A.L., Maruyama, K., Torchilin, V.P. and Huang, L. (1990). Amphiphathic polyethyleneglycols effectively prolong the circulation time of liposomes. FEBS Lett. 268:235-237.

Krishna, R. and Mayer, L.D. (2000). Multidrug resistance (MDR) in cancer: Mechanisms, reversal using modulators of MDR and the role of MDR modulators in intionfluenging the Dendrimers for pharmacokinetics of anticancer drugs. Eur J Pharm Sci. 11:265-283.

Kesharwani, P., Keerti, J. and Narendra K. J. (2014). Dendrimer as nanocarrier for drug delivery. Progress in Polymer Science 39: 268-307.

Kukowska-Latallo, J. F., Bielinska, A. U., Johnson, J., Spindler, R., Tomalia, D. A. and Baker, J. R. (1996). Efficient transfer of genetic material into mammalian cells by using Starburst. polyamidoamine dendrimers. Proc. Natl. Acad. Sci. 93:4897-4902.

Kojima, C., Kono, K., Maruyama, K. and Takagishi, T. (2000). Synthesis of polyamidoamine dendrimers having poly (ethylene glycol) grafts and their 
ability to encapsulate anticancer drugs. Bioconj Chem. 11:910-917.

Kono, K.., Liu, M. and Fréchet, J.M. (1999). Design of dendritic macromolecules containing folate or methotrexate residues. Bioconjugate Chemistry, 10:1115-1121.

Kihara, F., Arima, H., Tsutsumi, T., Hirayama, F. and Uekama, K. (2003). In-vitro and in-vivo gene transfer by an optimized alpha cyclodextrin conjugate with polyamidoamine Bioconjug Chem 14: 342-350.

Langer, R. (1990). New methods of drug delivery. Science, 249:1527- 1533.

Liu, M. Kono, K. and Frechet, J.M. (2000). Water-soluble dendritic unimolecular micelles: their potential as drug delivery agents. J. Control. Release, 65: $121-131$.

Links, M. and Brown, R. (1999). Clinical relevance of the molecular mechanisms of resistance to anticancer drugs. Expert Rev Mol Med. 199:1-21.

Martin, J., Roeck, J. and Ryder, P. (1985). A new class of polymers: Starburst ${ }^{\circledR}$ dendritic macromolecules. Polym. J. 17: 117- 132.

Malik ,N., Evagorou, E.G. and Duncan, R. (1999). Dendrimer platinate: A novel approach to cancer chemotherapy
Anticancer Drugs, 10:767-776.

Myc, A., Majoros, I. J., Thomas, T. P. and Baker, J. R. (2007). Dendrimer-based targeted delivery of an apoptotic sensor in cancer cells. Biomacromolecules, 8: 13-18.

Mintzer, M. A. and Grinstaff, M. W. (2011). Biomedical applications of dendrimers. Chem Soc Rev., 40:173-190.

Maeda, H., Wu, J., Sawa,T., Matsumura,Y. and Hori, K (2000). Tumor vascular permeability and the EPR effect in macromolecular therapeutics. J. Control. Release, 65: 271- 284.

Newkome, G.R. Moorefield, C.N. and Vogtle, F. (2001). Dendrimers and Dendrons:
Concepts, Syntheses, Applications, Wiley-VCH, Weinheim, Germany.

Ooya, T., Lee, J. and Park, K. (2004). Hydrotropic dendrimers of generations four and five: Synthesis, characterization, and hydrotropic solubilization of paclitaxel. Bioconjug Chem., 15: 1221-1229.

Ould-Ouali, L., Noppe, M., Langlois, X., Willems, B., and Te Riele, P. (2005). Self assembling PEG copolymers for oral delivery of poorly water soluble drugs: A case study with risperidone. Journal of Controlled Release, 102:657 - 668 .

Padilla De Jesus, O.L., Ihre, H.R., Gagne, L., Frechet, J.M. and Szoka, F.C. (2002). Polyester dendritic systems for drug delivery applications: in-vitro and invivo evaluation. Bioconjug. Chem. 13: 453- 461.

Patri, A.K., Kukowska-Latallo, J.F. and Baker, J.R. (2005). Targeted drug delivery with dendrimers: Comparison of the release kinetics of covalently conjugated drug and non-covalent drug inclusion complex. Adv. Drug Delivery Rev. 57:2203- 2214.

Pushkar, S., Philip, A., Pathak, K. and Pathak, D. $\quad$ (2006). Dendrimers:Nanotechnology Derived Novel Polymers in Drug Delivery. Indian J. Pharm. Educ. Res.,40: 153158.

Quintana, A., Raczka, E., Piehler, L., Lee, 1 ., Myc, A., Majoros, I., Patri, A..K., Thomas, T., Mule, J. and Baker, J.R. (2002). Design and function of a dendrimer based therapeutic nanodevice targeted to tumor cells through the folate receptor. Pharm Res, 19:1310- 1316.

Rajesh babu, V., Mallikarjun, V., Nikhat, S.R. and Srikanth, G. (2010). Dendrimers: A new carrier system for Drug Delivery. Int J Pharm and App Sci. 1:1-10.

Richter-Egger, D.L., Tesfai, A. and Tucker, S.A. (2001). Spectroscopic Investigation of poly(propyleneimine) dendrimers. Anal. Chem. 73:57435751. 
Saktivel, T., Toth, I. and Florence, A.T. (1998). Synthesis and physicochemical properties of lipophillic polyamide dendrimers. Pharm. res., 15: 776-782.

Sideratou, Z., Kontoyianni, C., Drossopoulou, G.I. and Paleos, C.M. (2010). Synthesis of a folate functionalized PEGylated poly (propylene imine) dendrimer as prospective targeted drug delivery system. Bioorg. Med. Chem. Lett., 20: 6513-6517.

Surendra T., Lipika B., and Malay, D. K. (2014). Dendrimer chemistry and host guest interactions for drug targeting. Int J Pharm Sci and Res, 5(1): 23202514.

Tack, F., Bakker, A,, Maes, S., Dekeyser, N. and Bruining, M. (2006). Modified poly(propylene imine) dendrimers as effective transfection agents for catalytic DNA enzymes (DNAzymes). J Drug Target, 14:69-86.

Twyman, L.J., Beezer, A.E., Esfand, R., Hardy, M.J. and Mitchell, J.C. (1999). The synthesis of water soluble dendrimers, and their application as possible drug delivery systems. Tetrahedron Lett. 40: 1743- 1746.

Tomalia, D.A., Baker, V., Dewald,-Hall, V.G. and Kallos, S. (2005). Birth of a new macromolecular architecture: Dendrimers as quantized building blocks for nanoscale synthetic

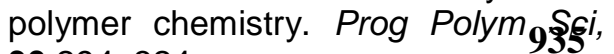
30:294-324.

Tripathi ,P.K., Khopade, A.J., Nagaich, S., Shrivastava, S., Jain, S., and Jain, N.K. (2002). Dendrimer grafts for delivery of 5-fluorouracil. Pharmazie, 57:261-264.

Wada, K., Arima, H., Tsutsumi, T., Chihara, Y., Hattori, K., and Uekama, K. (2005). Improvement of gene delivery mediated by mannosylated dendrimer/alpha cyclodextrin conjugates. J Control Release, 104:397-413.

Wang, Y., Guo, R., Cao, X., Shen, M., and Shi, X. (2011). Encapsulated of 2methoxyestradiol within multifunctional poly (amidoamine) dendrimers for targeted cancer therapy. Biomaterials, 32:3322-3329.

Wolinsky, J. B. and Grinstaff, M.W. (2008). Therapeutic and diagnostic applications of dendrimers for cancer treatment. Adv. Drug Deliv. Rev., 60: 1037-1055.

Yiyun, C., Zhenhua, $\mathrm{X}$., Minglu, $\mathrm{M}$ and Tonguen, X. (2008). Dendrimers as Drug Carriers: Applications in Different Routes of Drug. J. Pharma. Sci., 97: 123-143.

Zhou, Z., D'Emanuele, A. and Attwood, D. (2013). Solubility enhancement of paclitaxel using a linear-dendritic block copolymer. Int J Pharm., 452:173179. 